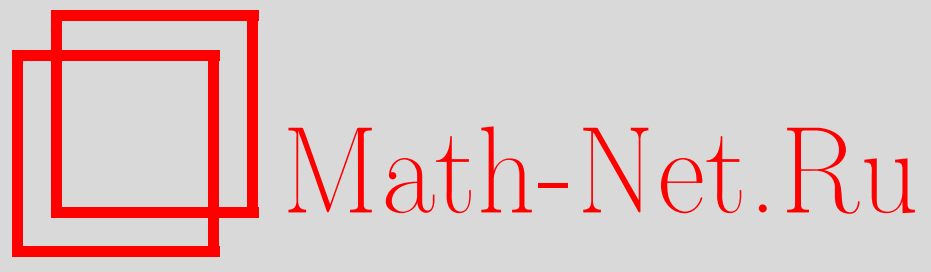

А. П. Лауринчикас, Д. Шяучюнас, Замечания об универсальности периодической дзета-функции, Матем. заметки, 2006, том 80, выпуск 4, 561-568

DOI: https://doi.org/10.4213/mzm2848

Использование Общероссийского математического портала Math-Net.Ru подразумевает, что вы прочитали и согласны с пользовательским соглашением http: //www . mathnet.ru/rus/agreement

Параметры загрузки:

IP : 18.234 .156 .22

26 апреля 2023 г., 04:10:55

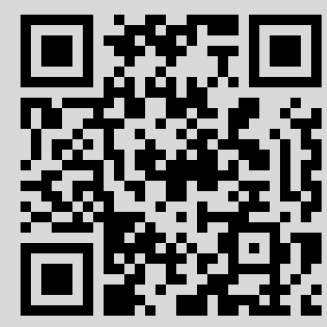




\section{ЗАМЕЧАНИЯ ОБ УНИВЕРСАЛЬНОСТИ ПЕРИОДИЧЕСКОЙ ДЗЕТА-ФУНКЦИИ}

\section{А. Лауринчикас, Д. Шяучюнас}

В статье рассматривается универсальность ряда Дирихле с периодическими коэффициентами. В случае мультипликативности коэффициентов получена универсальность этого ряда, а в общем случае получена универсальность в некотором множестве аналитических функций, связанном с вероятностным распределением.

Библиография: 7 названий.

1. Введение. Пусть, как обычно, $\mathbb{P}, \mathbb{N}, \mathbb{R}$ и $\mathbb{C}$ означают соответственно множества всех простых, натуральных, действительных и комплексных чисел. Через $\mathfrak{A}=\left\{a_{m}: m \in \mathbb{N}\right\}$ обозначим периодическую с наименьшим периодом $k \in \mathbb{N}$ последовательность комплексных чисел, т.е. $a_{m+k}=a_{m}$ для всех $m \in \mathbb{N}$. Пусть $s=\sigma+i t-$ комплексная переменная. Периодическая дзета-функция $\zeta(s ; \mathfrak{A})$ при $\sigma>1$ определяется рядом Дирихле

$$
\zeta(s ; \mathfrak{A})=\sum_{m=1}^{\infty} \frac{a_{m}}{m^{s}} .
$$

Легко видеть, что при $\sigma>1$

$$
\zeta(s ; \mathfrak{A})=\frac{1}{k^{s}} \sum_{l=1}^{k} a_{l} \zeta\left(s, \frac{l}{k}\right)
$$

где $\zeta(s, l / k)$ - дзета-функция Гурвица. Следовательно, последнее равенство дает аналитическое продолжение функции $\zeta(s ; \mathfrak{A})$ на всю комплексную плоскость, за исключением, быть может, точки $s=1$, с вычетом

$$
a=\frac{1}{k} \sum_{l=1}^{k} a_{l} .
$$

Если $a=0$, то $\zeta(s ; \mathfrak{A})$ является целой функцией.

Работа выполнена при поддержке Фонда науки и студий Литвы.

(C) А. ЛАуринчикас, Д. ШяУчюнас, 2006 
Штойдинг в [1] начал рассматривать универсальность в смысле Воронина (см. [2], $[3])$ функции $\zeta(s ; \mathfrak{A})$. Напоминаем, что открытое Ворониным свойство универсальности для дзета-функции Римана $\zeta(s)$ утверждает, что всякая аналитическая функция приближается сдвигами функции $\zeta(s)$. Точная формулировка теоремы Воронина следующая. Пусть $0<r<1 / 4$. Пусть $f(s)$ - функция, аналитическая внутри круга $|s| \leqslant r$ и непрерывная вплоть до границы этого круга. Если $f(s) \neq 0$ при $|s|<r$, то для всякого $\varepsilon>0$ существует $\tau=\tau(\varepsilon)$ такое, что

$$
\max _{|s| \leqslant r}\left|\zeta\left(s+\frac{3}{4}+i \tau\right)-f(s)\right|<\varepsilon .
$$

Через meas $\{A\}$ обозначим меру Лебега измеримого множества $A \subset \mathbb{R}$, и пусть при $T>0$

$$
\nu_{T}(\ldots)=\frac{1}{T} \operatorname{meas}\{\tau \in[0 ; T]: \ldots\},
$$

где вместо многоточия будем писать условие, которому удовлетворяет $\tau$. Тогда в [1] было получено следующее утверждение.

ТЕорема А. Предположим, что $k$ - нечетное простое число, $a_{m}$ не является кратным характера по модулю $k$ и $a_{k}=0$. Пусть $K$ - компактное подмножество полосы $D=\{s \in \mathbb{C}: 1 / 2<\sigma<1\}$, обладающее связным дополнением, а функиия $f(s)$ непрерывна на $K$ и аналитична внутри $K$. Тогда для всякого $\varepsilon>0$

$$
\liminf _{T \rightarrow \infty} \nu_{T}\left(\sup _{s \in K}|\zeta(s+i \tau ; \mathfrak{A})-f(s)|<\varepsilon\right)>0 .
$$

Цель настоящей заметки - изучить универсальность функции $\zeta(s ; \mathfrak{A})$ в общем случае. Напоминаем, что последовательность $\mathfrak{A}$ вполне мультипликативна, если $a_{1}=1$ и для всех $m, n \in \mathbb{N}$

$$
a_{m n}=a_{m} a_{n}
$$

и мультипликативна, если равенство (1) имеет место для всех взаимно простых $m$ и $n$. Через $p$ будем обозначать простое число.

ТЕОРема 1. Предположим, что последовательность $\mathfrak{A}$ вполне мультипликативна и

$$
\max _{p \leqslant k} \frac{\left|a_{p}\right|}{\sqrt{p}} \leqslant c_{1}<1 .
$$

Пусть $K$ - то же самое множество, что и в теореме $A$, а функиия $f(s)$ непрерывна, не имеет нулей в $K$ и аналитична внутри $K$. Тогда имеет место утверждение теоремы А.

ТЕорема 2. Предположим, что последовательность $\mathfrak{A}$ мультипликативна и для всех простых р выполняется оценка

$$
\sum_{\alpha=1}^{\infty} \frac{\left|a_{p^{\alpha}}\right|}{p^{\alpha / 2}} \leqslant c_{2}<1 .
$$

Пусть $K$ и $f(s)$ - те же самые, что и в теореме 1. Тогда имеет место утверждение теоремы $A$. 
Через $\gamma$ обозначим единичную окружность $\{s \in \mathbb{C}:|s|=1\}$ и определим

$$
\Omega=\prod_{p} \gamma_{p},
$$

где $\gamma_{p}=\gamma$ для всех простых $p$. Тогда $\Omega$ является компактной топологической абелевой группой. Пусть $\mathscr{B}(S)$ - класс борелевских множеств пространства $S$. Тогда на $(\Omega, \mathscr{B}(\Omega))$ может быть определена вероятностная мера Хаара $m_{H}$. Получаем вероятностное пространство $\left(\Omega, \mathscr{B}(\Omega), m_{H}\right)$. Через $\omega(p)$ обозначим проекцию элемента $\omega \in \Omega$ на координатное пространство $\gamma_{p}$ и для $m \in \mathbb{N}$ положим

$$
\omega(m)=\prod_{p^{\alpha} \| m} \omega^{\alpha}(p),
$$

где $p^{\alpha} \| m$ означает, что $p^{\alpha} \mid m$, но $p^{\alpha+1} \nmid m$. Таким образом, имеем, что $\omega(m)-$ вполне мультипликативная арифметическая функция. Пусть $H(D)$ - пространство аналитических в $D$ функций, наделенное топологией равномерной сходимости на компактах. Для $s \in D$ положим

$$
\zeta(s, \omega ; \mathfrak{A})=\sum_{m=1}^{\infty} \frac{a_{m} \omega(m)}{m^{s}} .
$$

Поскольку $\left|a_{m}\right|<c$ с некоторой постоянной $c>0$, стандартным путем (см., например, [4]) может быть доказано, что $\zeta(s, \omega ; \mathfrak{A})$ является $H(D)$-значным случайным элементом, определенным на вероятностном пространстве $\left(\Omega, \mathscr{B}(\Omega), m_{H}\right)$. Через $P_{\zeta}$ обозначим распределение случайного элемента $\zeta(s, \omega ; \mathfrak{A})$, т.е.

$$
P_{\zeta}(A)=m_{H}(\omega \in \Omega: \zeta(s, \omega ; \mathfrak{A}) \in A), \quad A \in \mathscr{B}(H(D)) .
$$

Пусть $S_{P_{\zeta}}-$ носитель меры $P_{\zeta} ; S_{P_{\zeta}}-$ это минимальное замкнутое множество пространства $H(D)$ такое, что $P_{\zeta}\left(S_{P_{\zeta}}\right)=1$.

Теорема 3. Предположим, что последовательность $\mathfrak{A}$ не является мультипликативной. Пусть $K$ - компактное подмножество полосы $D$, а функиия $f(s)$ принадлежит множеству $S_{P_{\zeta}}$. Тогда имеет место утверждение теоремы $A$.

2. Предельная теорема. Доказательства теорем 1-3 в отличии от теоремы А опираются на предельную теорему в смысле слабой сходимости вероятностных мер в пространстве $H(D)$ для функции $\zeta(s ; \mathfrak{A})$. Напомним, что если $P_{n}$ и $P-$ вероятностные меры на $(\Omega, \mathscr{B}(\Omega))$, то $P_{n}$ при $n \rightarrow \infty$ слабо сходится к $P$, если

$$
\lim _{n \rightarrow \infty} \int_{S} f \mathrm{~d} P_{n}=\int_{S} f \mathrm{~d} P
$$

для всякой действительной, непрерывной, ограниченной функции $f$. Определим вероятностную меру

$$
P_{T}(A)=\nu_{T}(\zeta(s+i \tau ; \mathfrak{A}) \in A), \quad A \in \mathscr{B}(H(D)) .
$$


Теорема 4. Вероятностная мера $P_{T}$ при $T \rightarrow \infty$ слабо сходится $\kappa P_{\zeta}$.

Мы дадим новое, более простое доказательство теоремы 4 без использования предельных теорем для полиномов Дирихле (см. ниже после леммы 2).

Пусть $\sigma_{1}>1 / 2$ - фиксированное число и для $m, n \in \mathbb{N}$

$$
v(m, n)=\exp \left\{-\left(\frac{m}{n}\right)^{\sigma_{1}}\right\} \text {. }
$$

Поскольку последовательность $\mathfrak{A}$ ограничена, нетрудно видеть, что ряд

$$
\zeta_{n}(s ; \mathfrak{A})=\sum_{m=1}^{\infty} \frac{a_{m} v(m, n)}{m^{s}}
$$

абсолютно сходится при $\sigma>1 / 2$. Действительно, пусть

$$
l_{n}(s)=\frac{s}{\sigma_{1}} \Gamma\left(\frac{s}{\sigma_{1}}\right) n^{s}, \quad n \in \mathbb{N},
$$

где $\Gamma(s)$ означает гамма-функцию Эйлера, и

$$
a_{n}(m)=\frac{1}{2 \pi i} \int_{\sigma_{1}-i \infty}^{\sigma_{1}+i \infty} \frac{l_{n}(s)}{s m^{s}} \mathrm{~d} s .
$$

В силу хорошо известных свойств гамма-функции

$$
a_{n}(m)=O\left(\frac{1}{m^{\sigma_{1}}} \int_{-\infty}^{\infty}\left|l_{n}\left(\sigma_{1}+i t\right)\right| \mathrm{d} t\right)=O\left(\frac{1}{m^{\sigma_{1}}}\right) .
$$

Следовательно, ряд

$$
\sum_{m=1}^{\infty} \frac{a_{m} a_{n}(m)}{m^{s}}
$$

абсолютно сходится при $\sigma>1 / 2$. Однако, применяя формулу Меллина

$$
\frac{1}{2 \pi i} \int_{b-i \infty}^{b+i \infty} \Gamma(s) a^{-s} \mathrm{~d} s=\mathrm{e}^{-a},
$$

справедливую для всех положительных $a$ и $b$, находим

$$
a_{n}(m)=\frac{1}{2 \pi i} \int_{\sigma_{1}-i \infty}^{\sigma_{1}+i \infty} \Gamma\left(\frac{s}{\sigma_{1}}\right)\left(\frac{m}{n}\right)^{-s} \mathrm{~d}\left(\frac{s}{\sigma_{1}}\right)=\exp \left\{-\left(\frac{m}{n}\right)^{\sigma_{1}}\right\}=v(m, n),
$$

что доказывает абсолютную сходимость при $\sigma>1 / 2$ ряда для $\zeta_{n}(s ; \mathfrak{A})$.

Определим

$$
Q_{T}(A)=\nu_{T}\left(\left\{p^{-i \tau}: p \in \mathbb{P}\right\} \in A\right), \quad A \in \mathscr{B}(\Omega) .
$$

Лемма 1. Вероятностная мера $Q_{T}$ при $T \rightarrow \infty$ слабо сходится $\kappa$ мере Хаapa $m_{H}$. 
ДокАЗАТЕЛЬСтво. Дуальной группой тора $\Omega$ является

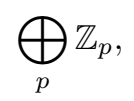

где $\mathbb{Z}_{p}=\mathbb{Z}$ для всякого $p \in \mathbb{P}$. Вектор $\boldsymbol{k}=\left(k_{2}, k_{3}, \ldots\right) \in \bigoplus_{p} \mathbb{Z}_{p}$, где только конечное число чисел $k_{p} \in \mathbb{Z}$ отличны от нуля, действует на $\Omega$ при помощи $\boldsymbol{x} \rightarrow \boldsymbol{x}^{\boldsymbol{k}}=$ $x_{2}^{k_{2}} x_{3}^{k_{3}} \cdots, \boldsymbol{x}=\left(x_{2}, x_{3}, \ldots\right) \in \Omega$. Поэтому преобразование Фурье $g_{T}(\boldsymbol{k})$ меры $Q_{T}$ имеет вид

$$
g_{T}(\boldsymbol{k})=\int_{\Omega} \prod_{p} x_{p}^{k_{p}} \mathrm{~d} Q_{T}=\frac{1}{T} \int_{0}^{T} \prod_{p} p^{-i \tau k_{p}} \mathrm{~d} \tau=\frac{1}{T} \int_{0}^{T} \exp \left\{-i \tau \sum_{p} k_{p} \log p\right\} \mathrm{d} \tau,
$$

где только конечное число целых чисел $k_{p}$ отличны от нуля. Поскольку система $\{\log p: p \in \mathbb{P}\}$ линейно независима над полем рациональных чисел, отсюда вытекает, что

$$
g_{T}(\boldsymbol{k})= \begin{cases}1, & \text { если } \boldsymbol{k}=\mathbf{0}, \\ \frac{1-\exp \left\{-i T \sum_{p} k_{p} \log p\right\}}{i T \sum_{p} k_{p} \log p}, & \text { если } \boldsymbol{k} \neq \mathbf{0} .\end{cases}
$$

Следовательно,

$$
\lim _{T \rightarrow \infty} g_{T}(\boldsymbol{k})= \begin{cases}1, & \text { если } \boldsymbol{k}=\mathbf{0}, \\ 0, & \text { если } \boldsymbol{k} \neq \mathbf{0},\end{cases}
$$

и лемма доказана.

Пусть $\widehat{\omega} \in \Omega$ и

$$
\zeta_{n}(s, \widehat{\omega} ; \mathfrak{A})=\sum_{m=1}^{\infty} \frac{a_{m} \widehat{\omega}(m) v(m, n)}{m^{s}},
$$

причем ряд, как и ряд $\zeta_{n}(s ; \mathfrak{A})$, абсолютно сходится при $\sigma>1 / 2$. На $(H(D)$, $\mathscr{B}(H(D)))$ определим две вероятностные меры

$$
P_{T, n}(A)=\nu_{T}\left(\zeta_{n}(s+i \tau ; \mathfrak{A}) \in A\right), \quad \widehat{P}_{T, n}(A)=\nu_{T}\left(\zeta_{n}(s+i \tau, \widehat{\omega} ; \mathfrak{A}) \in A\right) .
$$

Теорема 5. На $(H(D), \mathscr{B}(H(D)))$ существует вероятностная мера $P_{n}$ такая, что вероятностные меры $P_{T, n}$ и $\widehat{P}_{T, n}$ при $T \rightarrow \infty$ слабо сходятся $\kappa P_{n}$.

ДокАЗАтЕЛЬСтво. Определим функцию $u_{n}: \Omega \rightarrow H(D)$ формулой

$$
u_{n}(\omega)=\sum_{m=1}^{\infty} \frac{a_{m} \omega(m) v(m, n)}{m^{s}} .
$$

Тогда функция $u_{n}$ непрерывна; кроме того,

$$
u_{n}\left(\left\{p^{-i \tau}: p \in \mathbb{P}\right\}\right)=\zeta_{n}(s+i \tau ; \mathfrak{A}) .
$$

Следовательно, в силу леммы 1 и теоремы 5.1 из [5] получаем, что вероятностная мера $P_{T, n}$ при $T \rightarrow \infty$ слабо сходится к мере $m_{H} u_{n}^{-1}$. 
Теперь пусть функция $\widehat{u}_{n}: \Omega \rightarrow H(D)$ определена формулой

$$
\widehat{u}_{n}(\omega)=\sum_{m=1}^{\infty} \frac{a_{m} \widehat{\omega}(m) \omega(m) v(m, n)}{m^{s}} .
$$

Тогда аналогично случаю меры $P_{T, n}$ получаем, что вероятностная мера $\widehat{P}_{T, n}$ при $T \rightarrow \infty$ слабо сходится к мере $m_{H} \widehat{u}_{n}^{-1}$. Однако $\widehat{u}_{n}(\omega)=u_{n}(u(\omega))$, где $u(\omega)=\widehat{\omega} \omega$. Это и инвариантность меры Хаара $m_{H}$ влекут за собой равенство

$$
m_{H} \widehat{u}_{n}^{-1}=m_{H}\left(u_{n} u\right)^{-1}=\left(m_{H} u^{-1}\right) u_{n}^{-1}=m_{H} u_{n}^{-1},
$$

которое заканчивает доказательство теоремы.

Лемма 2. Пусть $K$ - компактное подмножество полосы D. Тогда

$$
\lim _{n \rightarrow \infty} \limsup _{T \rightarrow \infty} \frac{1}{T} \int_{0}^{T} \sup _{s \in K}\left|\zeta(s+i \tau ; \mathfrak{A})-\zeta_{n}(s+i \tau ; \mathfrak{A})\right| \mathrm{d} \tau=0
$$

и для почти всех $\omega \in \Omega$ в смысле мери $m_{H}$

$$
\lim _{n \rightarrow \infty} \limsup _{T \rightarrow \infty} \frac{1}{T} \int_{0}^{T} \sup _{s \in K}\left|\zeta(s+i \tau, \omega ; \mathfrak{A})-\zeta_{n}(s+i \tau, \omega ; \mathfrak{A})\right| \mathrm{d} \tau=0 .
$$

ДокАЗАТЕЛЬСтво. Поскольку при $\sigma>1 / 2$ имеет место оценка [6]

$$
\int_{1}^{T}|\zeta(\sigma+i t ; \mathfrak{A})|^{2} \mathrm{~d} t=O(T), \quad T \rightarrow \infty,
$$

доказательство леммы проводится стандартным путем, лишь в деталях отличающимся от случая дзета-функции Римана (см. [4; гл. 5]).

ДокаЗАТЕЛЬСтво тЕоремы 4. Теорема является следствием теоремы 5, леммы 2 и теоремы 4.2 из [5]. При этом применяются рассуждения, аналогичные используемым в доказательстве теоремы 5.1.8 из [4].

3. Доказательство теорем 1-3. Начнем с простого замечания о среднем значении чисел $\left|a_{p}\right|^{2}$. Пусть $\varphi(k)-$ функция Эйлера, а

$$
M(k)=\frac{1}{\varphi(k)} \sum_{\substack{l=1 \\(l, k)=1}}^{k}\left|a_{l}\right|^{2} .
$$

ЛЕмма 3. При $x \rightarrow \infty$

$$
\sum_{p \leqslant x}\left|a_{p}\right|^{2}=M(k) \frac{x}{\log x}(1+o(1)) .
$$

ДокАЗАТЕЛьство. В силу периодичности последовательности $\mathfrak{A}$ и теоремы о простых числах в арифметических прогрессиях находим, что при $x \rightarrow \infty$

$$
\sum_{p \leqslant x}\left|a_{p}\right|^{2}=\sum_{\substack{l=1 \\ l, k)=1}}^{k} \sum_{\substack{p \leqslant x \\ p \equiv l(\bmod k)}}\left|a_{p}\right|^{2}=\sum_{\substack{l=1 \\ l, k)=1}}^{k}\left|a_{l}\right|^{2} \sum_{\substack{p \leqslant x \\ p \equiv l(\bmod k)}} 1=M(k) \frac{x}{\log x}(1+o(1)) .
$$


ДокАЗАТЕЛЬСТво тЕОРЕмЫ 1. Поскольку последовальность $\mathfrak{A}$ вполне мультипликативна, случайный элемент $\zeta(s, \omega ; \mathfrak{A})$ может быть записан в виде

$$
\zeta(s, \omega ; \mathfrak{A})=\prod_{p}\left(1-\frac{a_{p} \omega(p)}{p^{s}}\right)^{-1},
$$

причем произведение для почти всех $\omega \in \Omega$ равномерно сходится на компактных подмножествах полосы $D$. Отсюда, используя условие теоремы, теорему 4 и лемму 3 , аналогично работе [7] получаем, что носителем меры $P_{\zeta}$ является множество

$$
\{g \in H(D): g(s) \neq 0 \text { или } g(s) \equiv 0\} .
$$

Поэтому дальнейшее доказательство полностью совпадает со случаем дзетафункции Римана (см. [4; теорема 6.5.2]).

ДОКАЗАТЕЛЬСТВО ТЕОРЕМЫ 2. В виду мультипликативности последовательности $\mathfrak{A}$, случайный элемент $\zeta(s, \omega ; \mathfrak{A})$ принимает вид

$$
\zeta(s, \omega ; \mathfrak{A})=\prod_{p}\left(1+\sum_{\alpha=1}^{\infty} \frac{a_{p^{\alpha} \omega^{\alpha}}(p)}{p^{\alpha s}}\right),
$$

причем произведение для почти всех $\omega \in \Omega$ равномерно сходится на компактных подмножествах полосы $D$. Поэтому условие теоремы, теорема 4 , лемма 3 и метод работы [7] опять позволяет получить, что носителем меры $P_{\zeta}$ является множество (2). Отсюда стандартным путем вытекает универсальность функции $\zeta(s ; \mathfrak{A})$.

ДоКАЗАТЕЛЬСТво ТЕОРЕМЫ 3. Поскольку последовательность $\mathfrak{A}$ немультипликативна, то случайный элемент $\zeta(s, \omega ; \mathfrak{A})$ не записывается в виде произведения. Поэтому мы должны рассматривать случайные величины $\omega(m), m \in \mathbb{N}$, которые не являются независимыми относительно меры $m_{H}$. Отсюда следует, что мы не можем воспользоваться тем вероятностным аппаратом, который действует в случае независимых случайных величин $\omega(p), p \in \mathbb{P}$. Поэтому остается ограничиться только условной версией универсальности функции $\zeta(s ; \mathfrak{A})$.

Определим множество

$$
G=\left\{g \in H(D): \sup _{s \in K}|g(s)-f(s)|<\varepsilon\right\} .
$$

Тогда множество $G$ открыто, и по условию теоремы оно является окрестностью элемента $f \in S_{P_{\zeta}}$. Носитель $S_{P_{\zeta}}$ меры $P_{\zeta}$ состоит из точек $x \in H(D)$ таких, что для каждой окрестности $O$ точки $x$ выполняется неравенство $P_{\zeta}(O)>0$. Таким образом, $P_{\zeta}(G)>0$. Поскольку в силу теоремы 4 и теоремы 2.1 из [5]

$$
\liminf _{T \rightarrow \infty} \nu_{T}\left(\sup _{s \in K}|\zeta(s+i \tau ; \mathfrak{A})-f(s)|<\varepsilon\right) \geqslant P_{\zeta}(G),
$$

то отсюда получаем утверждение теоремы.

ГипотеЗА. Если последовательность $\mathfrak{A}$ не мультипликативна, то $S_{P_{\zeta}}=H(D)$. 


\section{СПИСОК ЦИТИРОВАННОЙ ЛИТЕРАТУРЫ}

[1] J. Steuding, Value-distribution of L-functions and allied zeta-functions - with an emphasis on aspects of universality, Habilitationschrift, Frankfurt, J. W. Goethe-Universität, 2003.

[2] С. М. Воронин, "Теорема об "универсальности" дзета-функции Римана", Изв. АН СССР. Сер. матем., 39:1 (1975), 475-486.

[3] С. М. Воронин, А. А. Карацуба, Дзета-функиия Римана, Физматлит, М., 1994.

[4] A. Laurinčikas, Limit Theorems for the Riemann Zeta-Function, Kluwer, Dordrecht, 1996.

[5] П. Билингсли, Сходимость вероятностных мер, М., 1977.

[6] А. Лауринчикас, Д. Шяучюнас, "О периодической дзета-функции. II", Лит. матем. cб., 41:4 (2001), 461-476.

[7] A. Laurinčikas, R. Šleževičienè, "The universality of zeta-functions with multiplicative coefficients", Integral Transforms Special Functions, 13 (2002), 243-257.

\section{А. Лауринчикас}

Вильнюсский университет

E-mail: antanas.laurincikas@maf.vu.lt

\section{Д. Шяучюнас}

Шяуляйский университет

E-mail: siauciunas@fm.su.lt
Поступило

31.01 .2006 